\section{Psychophysical impact and optical and morphological characteristics of symptomatic non-advanced cataract}

S Charalampidou ${ }^{1,2}$, J Nolan ${ }^{3}$, J Loughman ${ }^{3,4}$, J Stack ${ }^{3}$, G Higgins ${ }^{1}$, L Cassidy ${ }^{5,6}$ and S Beatty ${ }^{1,3}$

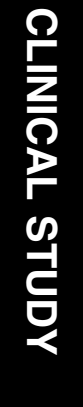

Institute of Eye Surgery and Institute of Vision Research, Whitfield Clinic, Waterford, Ireland

${ }^{2}$ Department of Postgraduate Studies, School of Medicine, Trinity College Dublin, Dublin, Ireland

${ }^{3}$ Macular Pigment Research Group, Waterford Institute of Technology, Waterford, Ireland Eye (2011) 25, 1147-1154; doi:10.1038/eye.2011.123 published online 10 June 2011

Keywords: psychophysical; symptomatic; non-advanced; cataract

\section{Introduction}

Age-related cataract is the most common eye disorder in the western world and, according to the World Health Organization, is one of the principal causes of blindness worldwide, therefore representing a major public-health issue. ${ }^{1-4}$ Not surprisingly, cataract surgery by phacoemulsification and intraocular lens (IOL) implantation is the most commonly performed surgical procedure worldwide. ${ }^{3,5-7}$

Valid evaluation of cataract and its visual consequences is indispensable for the purpose of investigating possible risk factors for cataract formation and for documenting progression of cataract in epidemiologic and cohort studies.

Also, reliable and valid assessment of cataract and its visual sequelae is equally important for clinical practice, if the ophthalmologist is to accurately evaluate the impact of age-related cataract on patient's visual performance and experience, and therefore identify those who are likely to benefit from surgical intervention. This is especially true in an era of falling thresholds for cataract surgery. ${ }^{8,9}$

Herman Snellen (1834-1908) developed a standardized test for visual acuity (VA) in
${ }^{4}$ Department of Optometry, School of Physics, Dublin Institute of Technology, Dublin, Ireland

${ }^{5}$ Department of Ophthalmology, Royal Victoria Eye and Ear Hospital, Dublin, Ireland

${ }^{6}$ Department of Ophthalmology, Trinity College Dublin, Dublin, Ireland

Correspondence:

S Charalampidou, Institute of Eye Surgery and Institute of Vision Research, Whitfield Clinic, Butlerstown North, Cork Road, Waterford, Ireland Tel: + 35387241 1425;

Fax: + 35351319849 .

E-mail: sonia.sofia1@ gmail.com

Received: 30 November 2010 Accepted in revised form: 8 April 2011

Published online: 10 June 2011

This study was presented at the Irish College of Ophthalmologists' Annual Conference, on the 30th of April 2010, in Dublin, Ireland and at the XVIII Congress of the European Society of Cataract and Refractive Surgeons, on the 7th of September 2010, in Paris, France. 
$1862 .^{10}$ This test is still widely used today and, in fact, represents the most frequently employed method of assessing visual function in cataract patients in clinical practice. ${ }^{11}$ The greatest limitation of using VA measurement as a means of assessing visual function rests on the fact that acuity is a measure of the resolving power of the eye, but in terms of contrast sensitivity (CS) function it is defined as the highest spatial frequency that can be detected at $100 \%$ contrast. ${ }^{12,13}$ However, the real world contains few visual stimuli at $100 \%$ contrast, but is full of visual stimuli of contrast lower than $100 \%$. Additionally, the well-lit environment of an ophthalmologist's office may not compare with the dimmer settings in which most individuals engage in daily activities. This, in turn, suggests that other (ie, non-acuity) measures of CS are better indicators of visual performance and experience for purposes of everyday life. ${ }^{14,15}$

The questionable ecological validity of VA charts hinders interpretation of patients' functional visual difficulty following the development of an ocular disease, such as age-related macular degeneration, ${ }^{16}$ cataract, glaucoma, and diabetic retinopathy. ${ }^{17}$

Consequently, it has been suggested that VA is not the most appropriate tool to investigate the impact of symptomatic non-advanced cataract on visual function and experience, ${ }^{11}$ and complementary and/or alternative techniques that have been investigated include $\mathrm{CS}^{18}$ glare disability (GD), ${ }^{19}$ reading performance, ${ }^{20}$ and functional visual difficulty assessed by various questionnaires. $^{21-23}$ Morphological and optical characteristics of lens opacification are also important, and can be assessed by various grading systems ${ }^{24}$ and Scheimpflug photography, ${ }^{25,26}$ respectively, and have the advantage of being independent of patient cooperation and ocular co-morbidity.

The purpose of this study was to investigate whether psychophysical, morphological, and/or optical measures of cataract are complementary to, or perhaps more appropriate than, corrected distance VA (CDVA) for the purposes of recording visual data that reflect subjective visual difficulty experienced by patients with cataract that exhibit relative sparing of high contrast acuity (0.4 logarithm of minimal angle of resolution (logMAR) or better).

\section{Materials and methods}

Consecutive patients with symptomatic non-advanced cataract and no other ocular pathology, scheduled to undergo phacoemulsification cataract extraction and implantation of the Tecnis 1-Piece (T1P) IOL (Advanced Medical Optics Inc., Santa Ana, CA, USA) for visual improvement, were recruited from the clinics taking place at a high-volume surgical practice between July 2009 and February 2010. For the purposes of this study, non-advanced cataract was defined as cataract presenting with CDVA better than or equal to 0.4 , as measured in the clinic using the logMAR chart provided by Test Chart $2000^{\mathrm{PRO}}$ (Thomson Software Solutions, Hatfield, UK) at a test distance of $4 \mathrm{~m}$. Only one eye of each patient (the one with the worse CDVA) was recruited. The fellow eyes of 65 of the 82 study eyes were phakic, whereas 17 were pseudophakic. Ethics committee approval was secured from the Local Regional Ethics Committee and the research was conducted in accordance with the principles laid down in the Declaration of Helsinki. Written and informed consent for participation in the study was obtained from all recruited patients before recruitment in the study.

Exclusion criteria were as follows: any preoperative ocular co-morbidity, a history of ocular trauma, diabetes mellitus (according to self-reported medical history; screening for diabetes mellitus was not performed for the purpose of this study;), and previous intraocular surgery.

Data were collected 2-4 weeks before planned cataract surgery and included: questionnaire score; CDVA; CS; GD; reading acuity and reading speed; stereoacuity; retinal sensitivity; lens optical density (LOD); and Lens Opacities Classification System (LOCS) III score.

\section{Subjective visual difficulty}

Subjectively perceived visual difficulty in everyday life was evaluated using the Priquest questionnaire. ${ }^{22}$ The questionnaire was self-administered, but the examiner was available to answer patients' queries in relation to any of the questionnaire items. A copy of the items in the Priquest questionnaire is given in Table 1. Rasch analysis was applied to the questionnaire data, thus calibrating item difficulty and patient ability on the same scale. $^{27,28}$

\section{Visual acuity}

CDVA was measured monocularly and with the patient's best subjective refraction, using the logMAR chart provided by Test Chart $2000^{\mathrm{PRO}}$ at a test distance of $4 \mathrm{~m}$.

\section{Contrast sensitivity}

CS was measured using both a letter chart (Test Chart $2000^{\mathrm{PRO}}$ ) and the sine-wave grating contrast test system: 'Functional Acuity Contrast Test' (FACT) (Optec 6500 Vision Tester, Stereo Optical Co., Inc., Chicago, IL, USA).

\section{Letter CS}

At a constant room illuminance of $8701 x$ and with distance correction on, each patient was asked to identify 
Table 1 Items in the Priquest questionnaire

Because of your vision, do you have difficulty with the following activities? (Response options: no difficulty, some difficulty, great difficulty, and extreme difficulty)

1. Reading newspaper print

2. Recognizing the faces of people you meet

3. Reading the prices of goods when you shop

4. Seeing to walk on uneven ground

5. Seeing to do needlework, etc

6. Reading TV text

7. Seeing to carry out a preferred activity/hobby

8. Do you feel that headlights, lamps, sunlight, or other lights dazzle you, reducing your vision?

9. Do you experience visual disturbances from differences (clarity, color, poor depth perception) between the two eyes?

10. If you have a job, does your present vision cause any problems?

11. If you are a car driver, does your present vision cause any difficulties in driving?

12. If you look after yourself or care for someone at home, does your present vision cause any problems?

Table 2 Contrast levels tested with letter chart

\begin{tabular}{lr}
\hline Contrast (\%) & CS \\
\hline 100 & 1.00 \\
70.8 & 1.41 \\
50.1 & 2.00 \\
35.5 & 2.82 \\
25.1 & 3.98 \\
17.8 & 5.62 \\
12.6 & 7.94 \\
8.9 & 11.24 \\
6.3 & 15.87 \\
4.5 & 22.22 \\
3.2 & 31.25 \\
2.2 & 45.45 \\
1.6 & 62.50 \\
1.1 & 90.91 \\
0.8 & 125.00 \\
0.6 & 166.67 \\
\hline
\end{tabular}

Abbreviation: CS, contrast sensitivity.

ETDRS letters in $\log$ MAR form, monocularly with the study eye, and at a distance of $4 \mathrm{~m}$. The contrast of letters of predetermined size (and therefore predetermined spatial frequency) was reduced incrementally using the software's contrast adjustment function until the contrast threshold of the patient was reached (ie, the patient could read no more letters). Table 2 shows the increments of decreasing contrast tested and their corresponding CS. Six different letter sizes (and corresponding spatial frequencies) were tested, each having the following angular subtense in cycles per degree (cpd): 6/120 letters (1.2 cpd), 6/60 letters ( $2.4 \mathrm{cpd}), 6 / 24$ letters $(6 \mathrm{cpd})$, 6/15 letters (9.6 cpd), 6/9.5 letters (15.16 cpd), and 6/6 letters (24cpd).

\section{Functional Acuity Contrast Test}

At a room illuminance of $1.5 \mathrm{~lx}$, monocularly with the study eye, and with distance correction, each patient was asked to look into the FACT. The patients were asked to look at five linear sine-wave grating charts of 1.5, 3, 6, 12, and $18 \mathrm{cpd}$, respectively. Each chart consisted of nine circular patches containing gratings of decreasing contrast, arranged in two rows (five patches above and four patches below). Patients were instructed to identify the orientation of the gratings by choosing 1 of 3 options: gratings tilted left ( +15 degrees), gratings upright (0 degrees), or gratings tilted right ( -15 degrees). Testing was performed under two different conditions: night (mesopic: 3 candelas $\left(\mathrm{cd} / \mathrm{m}^{2}\right)$ ) and day (photopic: $\left.85 \mathrm{~cd} / \mathrm{m}^{2}\right)$, in that order.

\section{Glare disability}

The FACT was repeated in a similar manner as performed to test CS, under mesopic and photopic conditions, in that order, but this time with additional glare light (1 lx for night and $10 \mathrm{~lx}$ for day glare testing).

\section{Reading performance}

Reading speed and near VA were measured with an English version of the standardized Radner Reading charts, the reliability and reproducibility of which have been established, both for subjects with normal eyesight and for those with visual difficulty. ${ }^{29}$

\section{Stereoacuity}

Stereoacuity was measured using the TNO stereo test (Lameris Instrumenten, Utrecht, Netherlands), an anaglyptic random dot test. The patients performed the quantitative part of the test (plates V-VII) and were awarded the stereoacuity of the smallest stereoacuity target that was correctly identified.

\section{Retinal sensitivity}

Retinal sensitivity was measured by performing microperimetry, with the Microperimeter MP 1 (Nidek Technologies Srl, Albignasego (PD), Italy). The patient's study eye was pharmacologically dilated with one drop of guttae Tropicamide BP $1 \% \mathrm{w} / \mathrm{v}$ minims (Chauvin Pharmaceuticals Ltd, Romford, UK) 20 min before the test. The central 10 degrees of fixation were examined. Retinal sensitivity was calculated for four areas: fixation (1 stimulus), within the central 5 degrees of fixation (average of 5 stimuli), between 5 and 10 degrees of 
fixation (average of 8 stimuli), and within 10 degrees of fixation (average of 13 stimuli).

\section{Lens optical density}

LOD was measured using Scheimpflug images taken by the Pentacam Comprehensive Eye Scanner (software version 1.16; Oculus Inc., Wetzlar, Germany), the repeatability and validity of which have been established. ${ }^{30}$ The study eye was pharmacologically dilated. Twenty-five images of the anterior segment of the eye were acquired. Density data were calculated by the software from the sum of the gray-scale values for the pixels along the axial length of the crystalline lens obtained from the cross-sectional image of the Scheimpflug camera. The density of the lens is standardized from 0 to 100 . Therefore, 0 means the lens shows no clouding, 100 means the lens is completely opaque. The gray scale of the individual Scheimpflug images provides the basis for objective quantification. The 'densitometry along a line' part of the software was used to analyze the images and a mean LOD value was recorded directly from the visual axis line appearing in the Scheimpflug image.

\section{LOCS III}

Cataracts were categorized and graded clinically at the slit lamp by a single ophthalmologist using the LOCS III. ${ }^{24}$ Cataracts are graded in terms of nuclear opalescence/nuclear color (1-6), cortical opacity (1-5), and posterior subcapsular opacity (1-5).

\section{Statistical analysis}

Statistical analysis was performed using the software package PASW Statistics 18.0 (IBM Corp., Somers, NY, USA). Descriptive statistics were calculated for all measured variables, including demographic, ocular, refractive, psychophysical, cataract optical and morphological data, as well as data on subjective functional visual difficulty (questionnaire).

Correlations between the different psychophysical measures of visual function, as well as optical characteristics of the studied cataracts, and Rasch-scaled questionnaire score were investigated using Spearman's rank correlation. Tests were two-sided. A one-way analysis of variance test with a Games-Howell post hoc analysis was used to compare the Rasch-scaled questionnaire score between the different cataract morphology (LOCS III) subgroups, and also between the different stereoacuity subgroups. Levene's test was used to test for equal variances. In all analyses, a $P$-value $<0.05$ was considered statistically significant.

\section{Results}

Eighty-two patients (82 eyes) met the inclusion criteria and were recruited into this study. The demographic, ocular, refractive, psychophysical, cataract optical and morphological data, as well as data on subjective visual difficulty, are given in Table 3.

We found statistically significant negative correlations between Rash-scaled Priquest score and mesopic GD (measured at 3 and $6 \mathrm{cpd} ; r=-0.396(P<0.01)$ and $-0.451(P<0.05)$, respectively) and between Rasch-scaled Priquest score and photopic GD (measured at 3 and $6 \mathrm{cpd} ; r=-0.328(P<0.01)$ and $-0.440(P<0.01)$, respectively).

We also report statistically significant positive correlations between preoperative Rasch-scaled Priquest score and CS by letters (measured at 1.2 and $2.4 \mathrm{cpd}$; $r=0.235(P<0.05)$ and $0.272(P<0.05)$, respectively $)$.

The questionnaire score was not related in a statistically significant way to any of the following: CDVA, photopic CS measured by letters (at any spatial frequency other than 1.2 and $2.4 \mathrm{cpd}$ ), mesopic or photopic CS measured by gratings (at any spatial frequency), mesopic GD (at any spatial frequency other than 3 and $6 \mathrm{cpd}$ ), photopic GD (at any spatial frequency other than 1.5 and $3 \mathrm{cpd}$ ), mean retinal sensitivity, reading acuity, and reading speed or LOD $(P>0.05$ for all).

Questionnaire score was not related in a statistically significant way to LOCS III nuclear opalescence/color score subgroup $(F(2,82)=0.63, P>0.05)$, LOCS III cortical score subgroup $(F(3,82)=0.62, P>0.05)$, or LOCS III subcapsular score subgroups $(F(2,80)=0.82, P>0.05$; see Table 4).

Stereoacuity subgroups did not differ in a statistically significant way in terms of questionnaire score $(F(5,78)=6.30, P>0.05$; see Table 5$)$.

\section{Discussion}

We carried out a study to evaluate a number of methods for assessing symptomatic non-advanced cataract and its visual consequences. The methods assessed included CDVA, photopic and mesopic CS (tested by letters (photopic only) and by gratings), photopic and mesopic GD (tested by gratings), reading performance (reading acuity and reading speed), stereoacuity, retinal sensitivity, LOD, and LOCS III. The purpose of this study was to investigate whether psychophysical, morphological, and/or optical measures of cataract are complementary to, or perhaps more appropriate than, CDVA for the purposes of documenting visual dissatisfaction attributable to cataract that exhibits relative sparing of high contrast acuity. 
Table 3 Demographic, ocular, refractive, psychophysical, cataract optical, cataract morphological and subjective visual difficulty data of patients with symptomatic non-advanced cataract and no other ocular pathology

\begin{tabular}{|c|c|c|c|c|}
\hline Variable & $n(\%)$ & Mean & $\pm S D$ & Range \\
\hline
\end{tabular}

\section{Demographic}

Age (years)

Gender

Male

Female

Ocular

Laterality

Right eye

Left eye

Refractive

SE study eye (D)

Psychophysical

CDVA study eye

CDVA fellow eye

LogCS by letters

(photopic)

Frequency (cpd)

1.2
2.4
6
9.6
15.16

24

LogCS by gratings

(mesopic conditions)

Frequency (cpd)

1.5
3
6
12
18

LogCS by gratings (photopic conditions)

Frequency (cpd)

1.5
3
6
12
18

$\log G D$

(mesopic conditions)

Frequency (cpd)

1.5
3
6
12
18

$82(100 \%) \quad 66.9 \quad 8.7 \quad 47$ to 85

$\begin{array}{llll}29(34 \%) & - & - & - \\ 57(66 \%) & - & - & -\end{array}$

$39(45 \%)--\quad-$

$47(55 \%) \quad-\quad-$

$82(100 \%)+0.162 .9-16.5$ to +6.5

$82(100 \%) \quad 0.18 \quad 0.16 \quad 0.62$ to -0.12 $82(100 \%) \quad 0.14 \quad 0.12 \quad 0.64$ to -0.14

$\begin{array}{llll}79(96 \%) & 1.38 & 0.19 & 0.90 \text { to } 1.66 \\ 79(96 \%) & 1.31 & 0.25 & 0.45 \text { to } 1.66 \\ 78(95 \%) & 1.00 & 0.31 & 0.15 \text { to } 1.49 \\ 72(88 \%) & 0.78 & 0.29 & 0.15 \text { to } 1.20 \\ 53(65 \%) & 0.54 & 0.27 & 0.15 \text { to } 1.05 \\ 13(16 \%) & 0.34 & 0.14 & 0.15 \text { to } 0.60\end{array}$

$\begin{array}{cccc}80(98 \%) & 1.44 & 0.22 & 0.85 \text { to } 2.00 \\ 77(94 \%) & 1.51 & 0.24 & 0.54 \text { to } 1.90 \\ 40(49 \%) & 1.31 & 0.20 & 1.08 \text { to } 1.65 \\ 5(6 \%) & 0.99 & 0.12 & 0.90 \text { to } 1.18 \\ 2(2 \%) & 0.69 & 0.12 & 0.60 \text { to } 0.78\end{array}$

$80(98 \%) \quad 1.35 \quad 0.0 .20 \quad 0.85$ to 1.85 $80(98 \%) \quad 1.57 \quad 0.22 \quad 1.00$ to 2.06 $57(70 \%) \quad 1.46 \quad 0.21 \quad 1.08$ to 2.11 $26(32 \%) \quad 1.14 \quad 0.17 \quad 0.90$ to 1.48 $20(24 \%) \quad 0.93 \quad 0.21 \quad 0.60$ to 1.23

$\begin{array}{cccc}64(78 \%) & 1.31 & 0.23 & 0.85 \text { to } 2.00 \\ 61(74 \%) & 1.45 & 0.22 & 1.00 \text { to } 1.90 \\ 23(28 \%) & 1.36 & 0.25 & 1.08 \text { to } 1.81 \\ 4(5 \%) & 1.04 & 0.16 & 0.90 \text { to } 1.18 \\ 3(4 \%) & 0.72 & 0.10 & 0.60 \text { to } 0.78\end{array}$

Table 3 (Continued)

\begin{tabular}{lcccc}
\hline Variable & $n(\%)$ & Mean & $\pm S D$ & Range \\
\hline $\begin{array}{l}\text { logGD } \\
\text { (photopic conditions) }\end{array}$ & & & & \\
Frequency (cpd) & & & & \\
1.5 & $75(91 \%)$ & 1.34 & 0.20 & 0.85 to 2.00 \\
3 & $72(88 \%)$ & 1.57 & 0.20 & 1.00 to 2.06 \\
6 & $47(57 \%)$ & 1.47 & 0.22 & 1.08 to 1.95 \\
12 & $23(28 \%)$ & 1.14 & 0.14 & 0.90 to 1.34 \\
18 & $13(16 \%)$ & 1.01 & 0.21 & 0.60 to 1.36 \\
LogRAD & $80(98 \%)$ & 0.33 & 0.21 & 0.1 to 1.1 \\
LogRAD score & $80(98 \%)$ & 0.34 & 0.21 & 0.1 to 1.1 \\
& & & & \\
Reading speed (wpm) & & & & \\
Letter size & & & & \\
1.2 & $80(98 \%)$ & 150 & 31 & 51 to 227 \\
1.1 & $80(98 \%)$ & 144 & 31 & 51 to 221 \\
0.9 & $77(94 \%)$ & 149 & 37 & 46 to 227 \\
0.8 & $77(94 \%)$ & 149 & 36 & 16 to 221 \\
0.7 & $77(94 \%)$ & 143 & 37 & 53 to 233 \\
0.6 & $72(88 \%)$ & 138 & 39 & 52 to 227 \\
0.5 & $71(87 \%)$ & 136 & 49 & 44 to 322 \\
0.4 & $69(84 \%)$ & 129 & 42 & 47 to 210 \\
0.3 & $56(68 \%)$ & 115 & 38 & 58 to 200 \\
0.2 & $35(43 \%)$ & 100 & 39 & 43 to 183
\end{tabular}

Stereoacuity (s of arc)

0
480
240
120
60
30
15

$\begin{array}{cccc}20(23.8 \%) & - & - & - \\ 5(6 \%) & - & - & - \\ 14(16.7 \%) & - & - & - \\ 30(35.7 \%) & - & - & - \\ 13(15.5 \%) & - & - & - \\ 2(2.4 \%) & - & - & - \\ 0(0 \%) & - & - & -\end{array}$

Mean retinal sensitivity $(d B)$

$\begin{array}{lllll}\text { Fixation } & 80(98 \%) & 14 & 5 & 0 \text { to } 20 \\ \text { Central } 5 \text { degrees } & 80(98 \%) & 16 & 4 & 0 \text { to } 20 \\ \begin{array}{l}\text { Between } 5 \text { and } \\ 10 \text { degrees }\end{array} & 80(98 \%) & 16 & 4 & 0 \text { to } 20 \\ \text { Central 10 degrees } & 80(98 \%) & 16 & 4 & 0 \text { to } 20\end{array}$

Cataract optical $(n=86)$

$\begin{array}{llrrc}\text { Average LOD } & 80(98 \%) & 12.4 & 1.7 & 9.8 \text { to } 18.2 \\ \text { Maximum LOD } & 80(98 \%) & 39.8 & 10.6 & 16.9 \text { to } 72.5 \\ \text { Minimum LOD } & 80(98 \%) & 5.5 & 1.1 & 2.4 \text { to } 12.7\end{array}$

Cataract morphological $(n=86)$

LOCS III nuclear score $80(98 \%) \quad 2.5 \quad 0.7 \quad 1$ to 4

LOCS III cortical score $80(98 \%) \quad 2.1 \quad 1.0 \quad 1$ to 4

LOCS III subcapsular $80(98 \%) \quad 1.4 \quad 0.8 \quad 1$ to 5

score

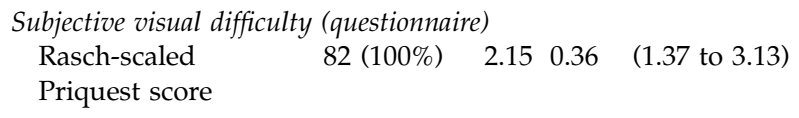

Abbreviations: CDVA, corrected-distance visual acuity; cpd, cycles per degree; CS, contrast sensitivity; $\mathrm{D}$, diopter; $\mathrm{dB}$, decibels; GD, glare disability; LOCS, lens opacities classification system; LOD, lens optical density; $\operatorname{logRAD}, \log$ reading acuity; SD, standard deviation; $\mathrm{SE}$, spherical equivalent; UDVA, unaided-distance visual acuity; wpd, words per minute. 
Table 4 Variation in subjective visual difficulty (questionnaire score) in different LOCS III classification subgroups

\begin{tabular}{|c|c|c|c|c|}
\hline \multirow[t]{2}{*}{ LOCS III classification } & \multicolumn{2}{|c|}{ Questionnaire score } & \multirow[t]{2}{*}{ F } & \multirow[t]{2}{*}{ P-value } \\
\hline & Mean & $\pm S D$ & & \\
\hline \multicolumn{5}{|c|}{ LOCS III nuclear opalescence/color score } \\
\hline 2 & 0.56 & 0.27 & & \\
\hline 3 & 0.51 & 0.29 & & \\
\hline 4 & 0.62 & 0.21 & $(2,82)=0.63$ & $>0.05$ \\
\hline \multicolumn{5}{|l|}{ LOCS III cortical score } \\
\hline 1 & 0.55 & 0.31 & & \\
\hline 2 & 0.53 & 0.26 & & \\
\hline 3 & 0.52 & 0.27 & & \\
\hline 4 & 0.68 & 0.24 & $(3,82)=0.62$ & $>0.05$ \\
\hline \multicolumn{5}{|c|}{ LOCS III subcapsular score } \\
\hline 1 & 0.56 & 0.27 & & \\
\hline 2 & 0.62 & 0.27 & & \\
\hline 3 & 0.44 & 0.33 & $(2,80)=0.82$ & $>0.05$ \\
\hline
\end{tabular}

Abbreviations: F, degrees of freedom; LOCS III, Lens Opacities Classification System III; SD, standard deviation.

Table 5 Variation in subjective visual difficulty (questionnaire score) in different stereoacuity subgroups

\begin{tabular}{lcccc}
\hline $\begin{array}{l}\text { Stereoacuity } \\
\text { (s of arc) }\end{array}$ & \multicolumn{2}{l}{ Questionnaire score } & $F$ & P-value \\
\cline { 2 - 3 } & Mean & $\pm S D$ & & \\
\hline 0 & 0.65 & 0.29 & & \\
480 & 0.57 & 0.36 & & \\
240 & 0.55 & 0.18 & & \\
120 & 0.48 & 0.28 & & \\
60 & 0.48 & 0.29 & $(5,78)=6.30$ & $>0.05$
\end{tabular}

Abbreviations: $F$, degrees of freedom; SD, standard deviation.

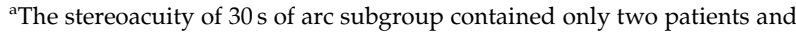
was therefore excluded from the above analysis due to its small size.

There was no statistically significant correlation between CDVA of the study eye and subjective visual difficulty in our study. This finding is consistent with the authors' suspicion that, in the context of symptomatic non-advanced cataract, high contrast VA, in isolation, is not an appropriate psychophysical test to evaluate the visual impact of the disease process, and the importance of this finding rests on the fact that CDVA is still the most commonly used psychophysical test in the assessment of cataract and its visual sequelae.

However, there was a statistically significant negative correlation between subjective visual difficulty (as measured by the Rasch-scaled questionnaire score) and mesopic GD (measured at 3 and $6 \mathrm{cpd}$ ) and photopic GD (measured at 3 and $6 \mathrm{cpd}$ ). This is an important clinical finding because it suggests that, for cataracts with little or only marginal impact on high contrast VA, mesopic and photopic GD measured at medium spatial frequencies is a more valid psychophysical tool than CDVA, and one that better reflects subjective visual difficulty attributable to the lens opacity.

The data also suggest that a value of mesopic GD at 3 cpd of $<29$ (corresponding to inability to correctly identify the fourth gratings patch presented during the test), a value of mesopic GD at 6 cpd of $<23$ (corresponding to inability to correctly identify the third gratings patch presented during the test), a value of photopic GD at $3 \mathrm{cpd}$ of $<40$ (corresponding to inability to correctly identify the fifth gratings patch presented during the test), and a value of photopic GD at $6 \mathrm{cpd}$ of $<33$ (corresponding to inability to correctly identify the fourth gratings patch presented during the test) are good indicators of subjective visual difficulty caused by cataract that is relatively sparing of high contrast acuity. In other words, if a patient with lenticular opacity and good high contrast CDVA complains of visual difficulty in the absence of ocular co-morbidity, and yet cannot correctly identify the presented gratings patches during GD testing, at the aforementioned levels of CS, then the lenticular opacity is indeed likely to be the cause of his/ her subjective visual difficulty. Of note, it is perhaps unsurprising that symptomatic non-advanced cataracts are best judged by a test which represents a function of light scatter, reduced retinal illumination and blur effect of cataract, such as mesopic and photopic GD at medium spatial frequencies. It is well known that developing cataracts cause increased intraocular forward light scatter, which causes visual difficulty due to loss of retinal image contrast, and this type of visual difficulty is called GD. ${ }^{19,31}$ It has also been previously reported that the amount of scatter can be evaluated indirectly by measuring GD, ${ }^{32}$ that GD offers a discriminative and valid measure of visual assessment in cataract, ${ }^{33}$ including cataract with good high contrast VA in the presence of functional complaints, ${ }^{34,35}$ that GD improves after cataract surgery, ${ }^{36}$ and that this improvement is independent of improvement in high contrast acuity. ${ }^{37}$ We suggest that testing mesopic and photopic GD at a medium spatial frequencies will help assess symptomatic non-advanced cataracts. The clinical test we suggest is both user- and clinic-friendly requires no more patient cooperation than a standard VA test, and consumes a considerably shorter amount of time ( $\sim 3 \mathrm{~min})$.

Interestingly, there was no statistically significant correlation between questionnaire score and average LOD. This finding suggests that LOD is unlikely to represent an objective surrogate or alternative to psychophysical testing in an eye with cataract that has little impact on high contrast VA.

Also, there were no statistically significant differences in terms of questionnaire score between different 
LOCS III nuclear opalescence/color score subgroups, cortical score subgroups or subcapsular score subgroups, indicating that this grading system of cataract morphology (LOCS III) is unlikely to represent a valid means of documenting the lens opacity in a way that reflects the patients' symptoms attributable to the cataract, in cases where there is relative sparing of high contrast acuity.

We report a lack of correlation between subjective visual difficulty and retinal sensitivity, indicating that this test is also unlikely to represent a useful means of recording visual data that reflect subjective visual difficulty in patients with symptomatic non-advanced cataract.

Stereoacuity, in our study, was not found to be indicative of overall subjective visual difficulty attributable to the lens opacity. However, it should be borne in mind that the TNO stereotest, used here, requires excellent fusion and is also known to be adversely affected by increasing age in normal individuals who report no difficulty in judging distances in everyday tasks. ${ }^{38}$ In other words, our findings may indicate that stereoacuity is a psychophysical test which is indeed affected by symptomatic non-advanced cataract (reflected in the observation that the vast majority of patients (over $82 \%$ ) had stereoacuity of $120 \mathrm{~s}$ of arc or less, and over $23 \%$ of patients had unmeasurable levels of stereoacuity), but may not be a good reflection of subjective visual difficulty in the presence of good CDVA in the fellow eye.

\section{Conclusion}

In conclusion, this study demonstrates that symptomatic non-advanced cataract, in the presence of good high contrast CDVA and in the absence of coexisting ocular pathology, is associated with subjective functional visual difficulty (measurable by a validated questionnaire), which is best reflected in a decrease in mesopic and photopic GD measured at medium spatial frequencies. High contrast VA does not reflect the patient's visual dissatisfaction in such cases. Ophthalmologists should consider testing mesopic and/or photopic GD at a medium spatial frequency to quantify the impact of symptomatic non-advanced cataract in those patients who report functional visual difficulty, not reflected in their high contrast VA test result. This clinic- and userfriendly test requires no more patient cooperation than a standard high contrast VA test, and takes $<3$ min to conduct. The collection of such preoperative data will represent a valid record of adversely affected vision where standard high contrast VA may be essentially normal or near normal, and will inform professional practice by assisting ophthalmologists and their patients in deciding the best time for cataract surgery, an issue which is becoming increasingly important in an era of falling thresholds for this procedure.

\section{Summary}

\section{What was known before}

- It has been suggested that visual acuity is not the most appropriate tool to investigate the impact of symptomatic non-advanced cataract on visual function and experience, and complimentary and/or alternative techniques that have been investigated include contrast sensitivity, glare disability, reading performance, and functional visual difficulty assessed by various questionnaires.

\section{What this study adds}

- Our research shows that symptomatic non-advanced cataracts are associated with subjective visual difficulty (measurable by a validated questionnaire), best reflected in a decrease in mesopic and photopic contrast sensitivity under conditions of glare, at medium spatial frequencies.

\section{Conflict of interest}

The authors declare no conflict of interest.

\section{Acknowledgements}

Supported in part by a grant by Abbott Medical Optics, Santa Ana, CA, USA.

\section{References}

1 Klein BE, Klein R, Lee KE. Incidence of age-related cataract over a 10-year interval: the Beaver Dam Eye Study. Ophthalmology 2002; 109(11): 2052-2057.

2 Congdon N, Vingerling JR, Klein BE, West S, Friedman DS, Kempen J et al. Prevalence of cataract and pseudophakia/ aphakia among adults in the United States. Arch Ophthalmol 2004; 122(4): 487-494.

3 Etzioni DA, Liu JH, Maggard MA, Ko CY. The aging population and its impact on the surgery workforce. Ann Surg 2003; 238(2): 170-177.

4 Thylefors B. The World Health Organization's programme for the prevention of blindness. Int Ophthalmol 1990; 14(3): 211-219.

5 Hodge W, Horsley T, Albiani D, Baryla J, Belliveau M, Buhrmann $\mathrm{R}$ et al. The consequences of waiting for cataract surgery: a systematic review. CMAJ 2007; 176(9): 1285-1290.

6 Lavanya R, Wong TY, Aung T, Tan DT, Saw SM, Tay WT et al. Prevalence of cataract surgery and post-surgical visual outcomes in an urban Asian population: the Singapore Malay Eye Study. Br J Ophthalmol 2009; 93(3): 299-304.

7 Panchapakesan J, Rochtchina E, Mitchell P. Five-year change in visual acuity following cataract surgery in an older community: the Blue Mountains Eye Study. Eye (Lond) 2004; 18(3): 278-282.

8 Chu RY, Taney-Rostov AR. Cataract surgery: new options, new challenges. Rev Ophthalmol 2006; 13(7): 71-78. 
9 Black N, Browne J, van der Meulen J, Jamieson L, Copley L, Lewsey J. Is there overutilisation of cataract surgery in England? Br J Ophthalmol 2009; 93(1): 13-17.

10 Snellen H. Test-Types for Determination of the Acuteness of Vision [Probebuchstaben zur Betimmung der Sehscharfe]. PW van de Weijer: Utrecht, 1862.

11 Jack KJ. Clinical Ophthalmology, A Systematic Approach. 5th ed. Elsevier: Oxford, UK, 2005, p 343.

12 Neelam K, Nolan J, Chakravarthy U, Beatty S. Psychophysical function in age-related maculopathy. Surv Ophthalmol 2009; 54(2): 167-210.

13 Owsley C. Contrast sensitivity. Ophthalmol Clin North Am 2003; 16(2): 171-177.

14 Evans DW, Ginsburg AP. Contrast sensitivity predicts agerelated differences in highway-sign discriminability. Hum Factors 1985; 27(6): 637-642.

15 Ginsburg AP, Evans DW, Sekule R, Harp SA. Contrast sensitivity predicts pilots' performance in aircraft simulators. Am J Optom Physiol Opt 1982; 59(1): 105-109.

16 Dubuc S, Wittich W, Gomolin JE, Kapusta M, Overbury O. Beyond visual acuity: functional outcome and patient satisfaction following treatment for age-related macular degeneration. Can J Ophthalmol 2009; 44(6): 680-685.

17 Broman AT, Munoz B, Rodriguez J, Sanchez R, Quigley HA, Klein $\mathrm{R}$ et al. The impact of visual impairment and eye disease on vision-related quality of life in a MexicanAmerican population: proyecto VER. Invest Ophthalmol Vis Sci 2002; 43(11): 3393-3398.

18 Stifter E, Sacu S, Thaler A, Weghaupt H. Contrast acuity in cataracts of different morphology and association to selfreported visual function. Invest Ophthalmol Vis Sci 2006; 47(12): 5412-5422.

19 Aslam TM, Haider D, Murray IJ. Principles of disability glare measurement: an ophthalmological perspective. Acta Ophthalmol Scand 2007; 85(4): 354-360.

20 Stifter E, Sacu S, Benesch T, Weghaupt H. Impairment of visual acuity and reading performance and the relationship with cataract type and density. Invest Ophthalmol Vis Sci 2005; 46(6): 2071-2075.

21 Stifter E, Sacu S, Weghaupt H. Functional vision with cataracts of different morphologies: comparative study. J Cataract Refract Surg 2004; 30(9): 1883-1891.

22 Lundstrom M, Albrecht S, Hakansson I, Lorefors R, Ohlsson S, Polland W et al. NIKE: a new clinical tool for establishing levels of indications for cataract surgery. Acta Ophthalmol Scand 2006; 84(4): 495-501.

23 Lundstrom M, Pesudovs K. Catquest-9SF patient outcomes questionnaire: nine-item short-form Rasch-scaled revision of the Catquest questionnaire. J Cataract Refract Surg 2009; 35(3): 504-513.

24 Chylack Jr LT, Wolfe JK, Singer DM, Leske MC, Bullimore MA, Bailey IL et al. The Lens Opacities
Classification System III. The Longitudinal Study of Cataract Study Group. Arch Ophthalmol 1993; 111(6): 831-836.

25 Pei X, Bao Y, Chen Y, Li X. Correlation of lens density measured using the Pentacam Scheimpflug system with the Lens Opacities Classification System III grading score and visual acuity in age-related nuclear cataract. $\mathrm{Br}$ J Ophthalmol 2008; 92(11): 1471-1475.

26 Grewal DS, Brar GS, Grewal SP. Correlation of nuclear cataract lens density using Scheimpflug images with Lens Opacities Classification System III and visual function. Ophthalmology 2009; 116(8): 1436-1443.

27 Wright BD, Masters GN. Rating Scale Analysis (Rasch Measurement Book. Messa Press: Chicago, IL, 1982.

28 Wright BD, Stone MH. Best Test Design; Rasch Measurement. MESA Press: Chicago, IL, 1979.

29 Stifter E, Konig F, Lang T, Bauer P, Richter-Muksch S, Velikay-Parel $\mathrm{M}$ et al. Reliability of a standardized reading chart system: variance component analysis, test-retest and inter-chart reliability. Graefes Arch Clin Exp Ophthalmol 2004; 242(1): 31-39.

30 Kirkwood BJ, Hendicott PL, Read SA, Pesudovs K. Repeatability and validity of lens densitometry measured with Scheimpflug imaging. J Cataract Refract Surg 2009; 35(7): 1210-1215.

31 Babizhayev MA, Minasyan H, Richer SP. Cataract halos: a driving hazard in aging populations. Implication of the Halometer DG test for assessment of intraocular light scatter. Appl Ergon 2009; 40(3): 545-553.

32 Elliott DB. Evaluating visual function in cataract. Optom Vis Sci 1993; 70(11): 896-902.

33 Elliott DB, Bullimore MA. Assessing the reliability, discriminative ability, and validity of disability glare tests. Invest Ophthalmol Vis Sci 1993; 34(1): 108-119.

34 Superstein R, Boyaner D, Overbury O, Collin C. Glare disability and contrast sensitivity before and after cataract surgery. J Cataract Refract Surg 1997; 23(2): 248-253.

35 Superstein R, Boyaner D, Overbury O. Functional complaints, visual acuity, spatial contrast sensitivity, and glare disability in preoperative and postoperative cataract patients. J Cataract Refract Surg 1999; 25(4): 575-581.

36 Hard AL, Beckman C, Sjostrand J. Glare measurements before and after cataract surgery. Acta Ophthalmol (Copenh) 1993; 71(4): 471-476.

37 Rubin GS, Adamsons IA, Stark WJ. Comparison of acuity, contrast sensitivity, and disability glare before and after cataract surgery. Arch Ophthalmol 1993; 111(1): 56-61.

38 Garnham L, Sloper JJ. Effect of age on adult stereoacuity as measured by different types of stereotest. $\mathrm{Br} J$ Ophthalmol 2006; 90(1): 91-95. 